\title{
IRMPD Spectroscopy of Homo- and Heterochiral Asparagine Proton-Bound Dimers in the Gas Phase
}

\author{
Åke Andersson, ${ }^{\dagger}$ Mathias Poline, ${ }^{\ddagger}$ Kas J. Houthuijs,, Rianne E. van Outersterp, \\ Giel Berden, $₫$ Jos Oomens, "and Vitali Zhaunerchyk*, ${ }^{*}$ \\ $\dagger$ Department of Physics, University of Gothenburg, Gothenburg, Sweden \\ $\ddagger$ Department of Physics, Stockholm University, Stockholm, Sweden \\ \Radboud University, Institute for Molecules and Materials, FELIX Laboratory, \\ Toernooiveld 7, 6525ED, Nijmegen, The Netherlands \\ E-mail: vitali.zhaunerchyk@physics.gu.se
}




\section{Supporting Information for "IRMPD Spectroscopy of Homo- and Heterochiral Asparagine Proton-Bound Dimers in the Gas Phase"}

\section{$1.1 \quad$ Experimental spectra}

Figure S1 - S3 show the experimental IRMPD spectra in greater detail. The intensities are the strongest in the range of $1000-1900 \mathrm{~cm}^{-1}$, as indicated by the grid.

\subsection{Calculated energies}

Figure S4 shows calculated energies using different methods. For each the structure is optimized with B3LYP-GD3BJ, $\omega$ B97XD, or M06-2X using the 6-311 $++\mathrm{G}^{* *}$ basis set. G4MP2 is then employed for a single-point calculation.

The results show that while electronic energy is not dependent on the choice of optimization method, Gibbs energy is. As an extreme example, the energy difference between DD-A1 and DD-B1 is $-2.358,3.258$, or $6.157 \mathrm{~kJ} \mathrm{~mol}^{-1}$ when B3LYP-GD3BJ, $\omega$ B97XD, or M06-2X is used. For comparison, at room temperature $k_{\mathrm{B}} T=2.494 \mathrm{~kJ} \mathrm{~mol}^{-1}$, which means that the relative abundances of conformers strongly depend on choice of method.

Table 1 lists the relative electronic energies $E$, Gibbs energies $G$, and abundances $p$ of conformers. 


\subsection{Figures and Tables}

Experimental IRMPD spectra of asparagine dimers.

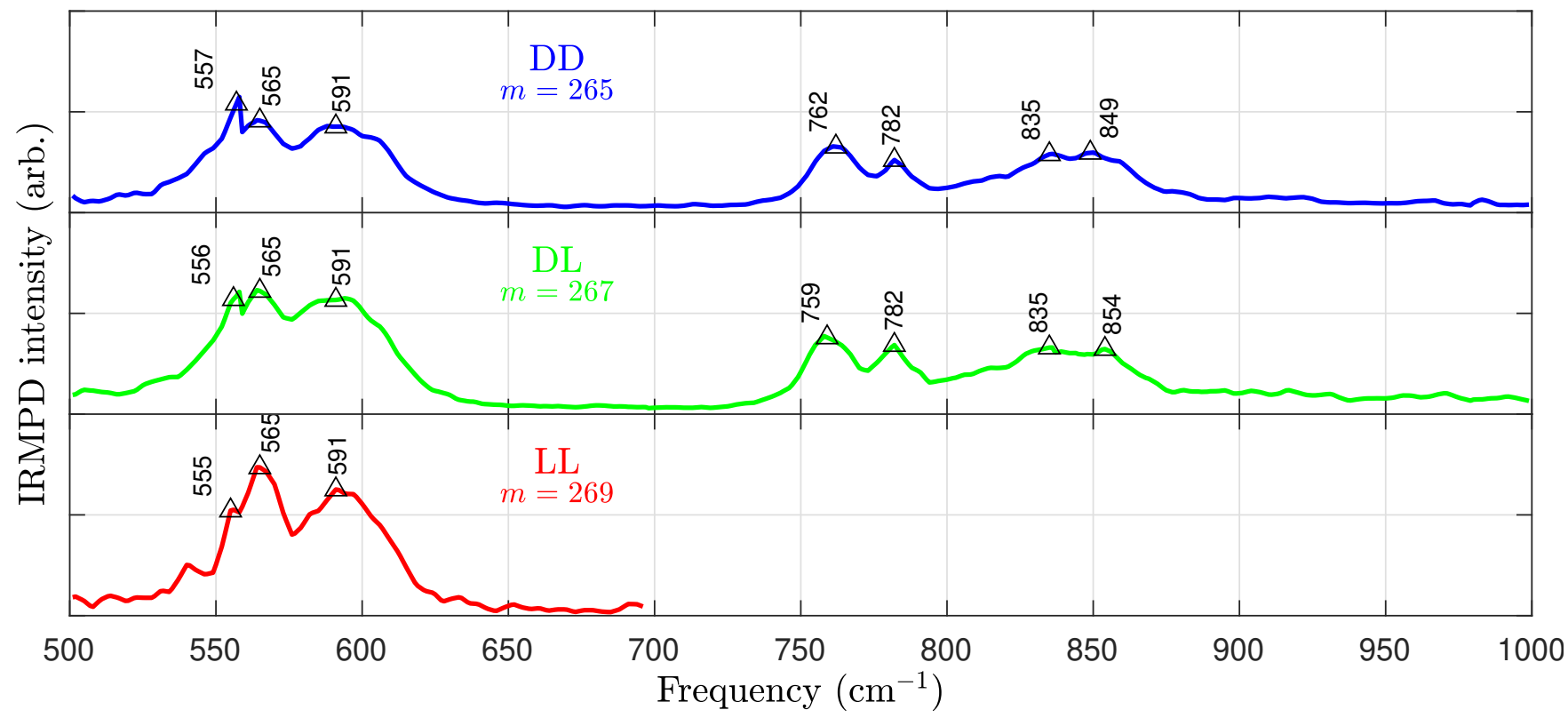

Figure S1: Experimental IRMPD spectra in the range of $500-1000 \mathrm{~cm}^{-1}$. 
Experimental IRMPD spectra of asparagine dimers.

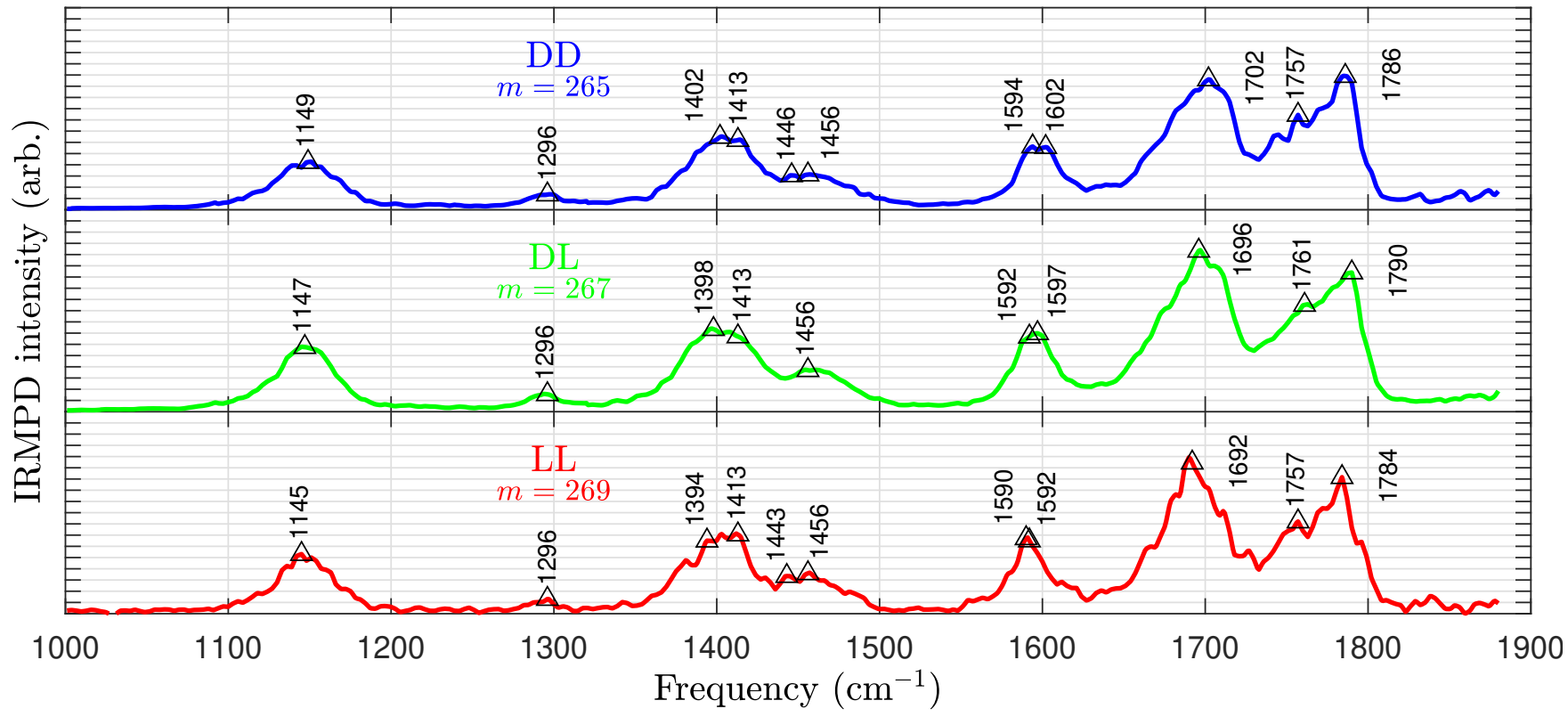

Figure S2: Experimental IRMPD spectra in the range of $1000-1900 \mathrm{~cm}^{-1}$.

Experimental IRMPD spectra of asparagine dimers.

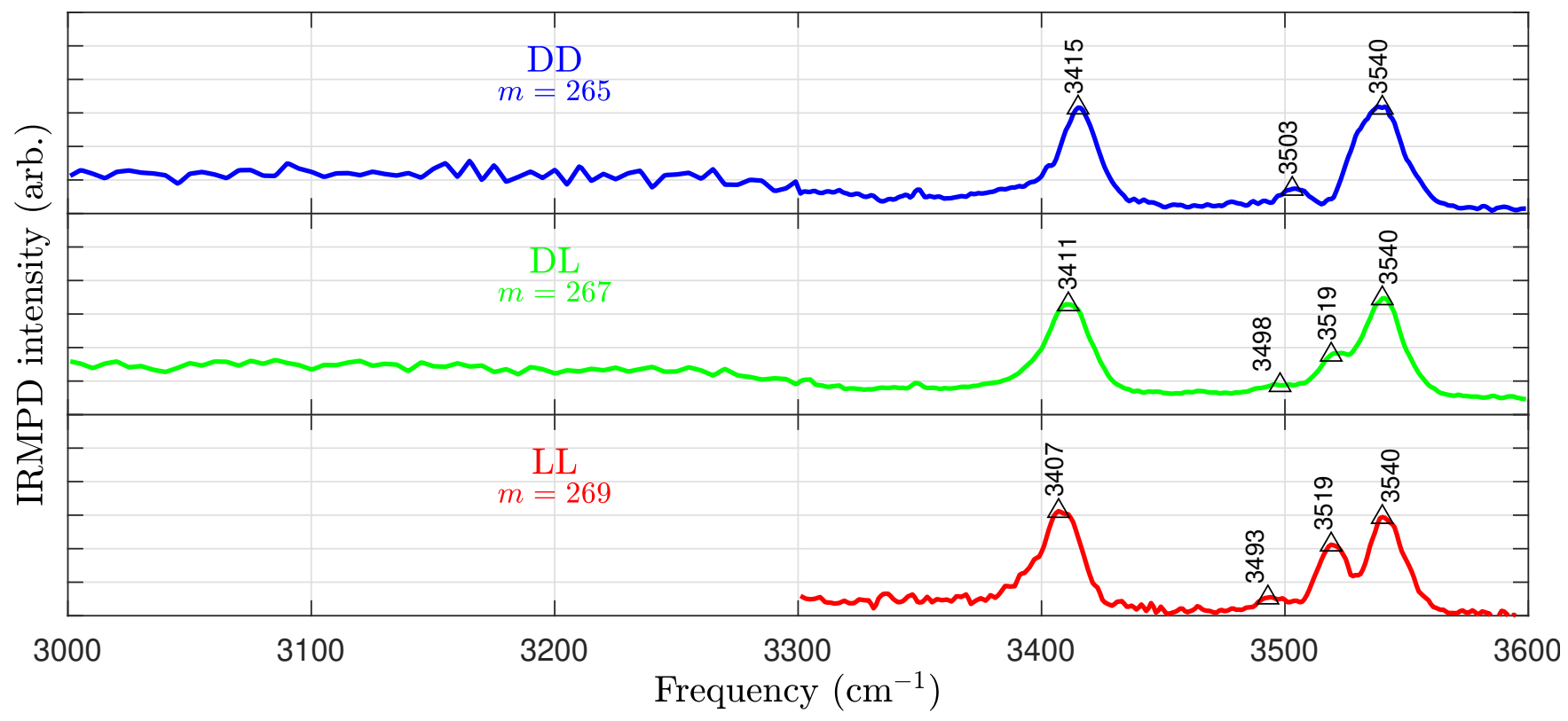

Figure S3: Experimental IRMPD spectra in the range of $3000-3600 \mathrm{~cm}^{-1}$. 


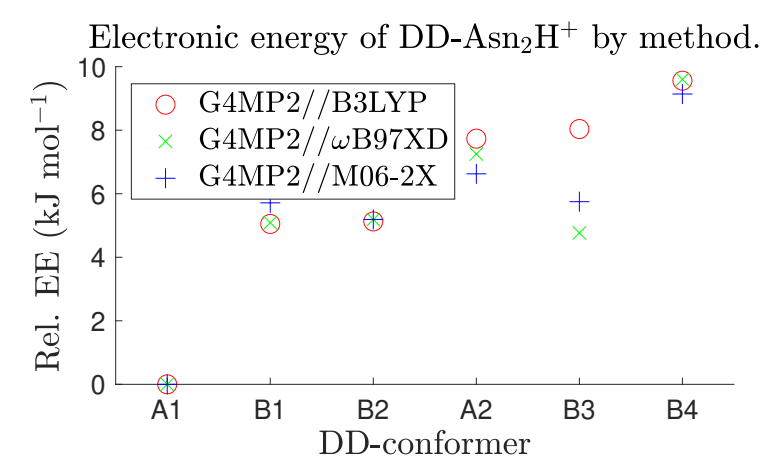

Electronic energy of DL-Asn ${ }_{2} \mathrm{H}^{+}$by method.
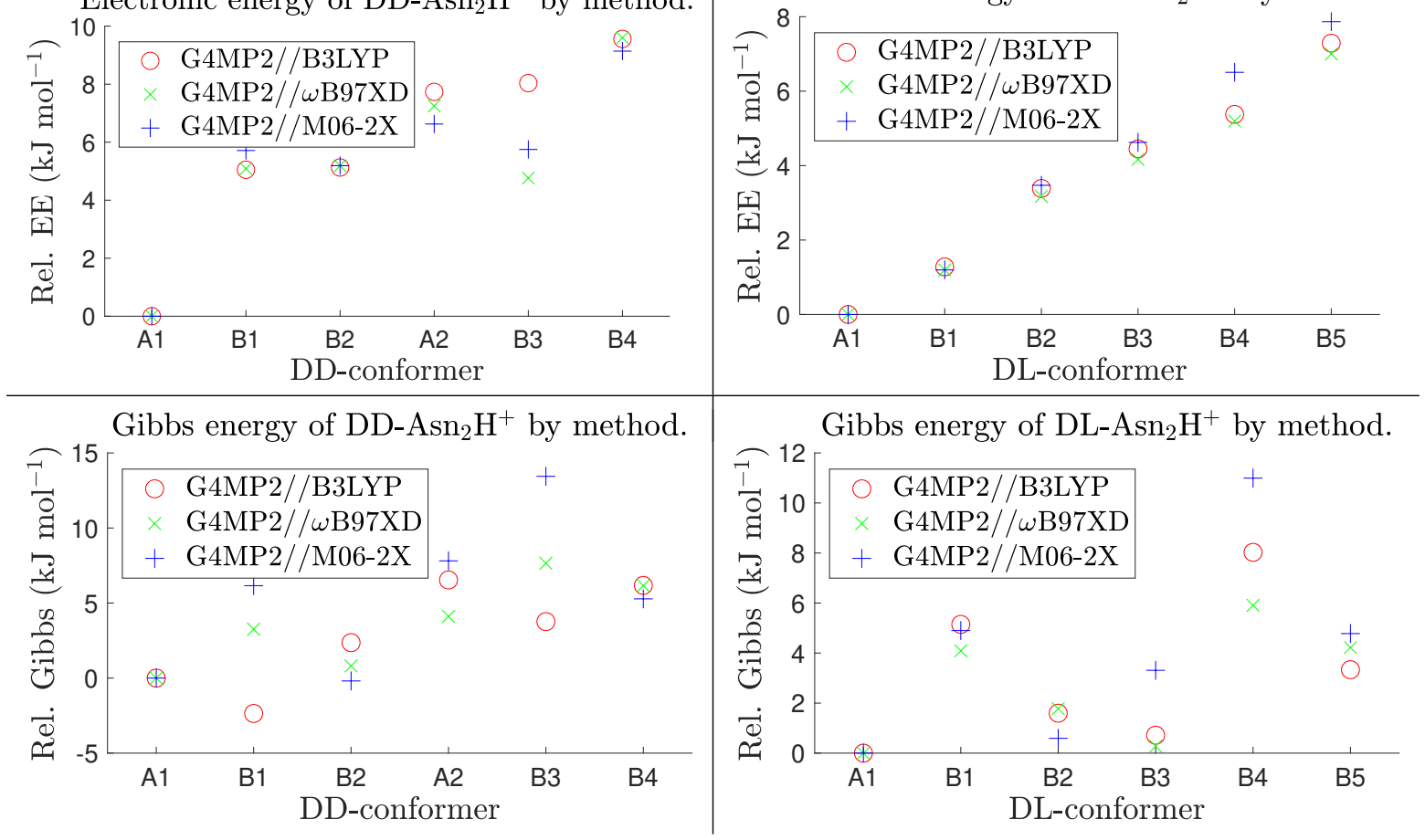

Figure S4: Computed relative (top) electronic and (bottom) Gibbs energies of the most stable (left) DD-Asn $\mathrm{H}^{+}$and (right) DL- $\mathrm{Asn}_{2} \mathrm{H}^{+}$conformers using three different methods. The 3D-structure of each conformer is optimized with one of B3LYP-GD3BJ, $\omega$ B97XD, or M06-2X. G4MP2 is then employed for a single-point calculation. In all calculations the basis set is $6-311++\mathrm{G}^{* *}$. 
Table 1: Relative electronic and Gibbs energies of the most stable conformers in $\mathrm{kJ} \mathrm{mol}^{-1}$, calculated with B3LYP-GB3BJ/6-311 $++\mathrm{G}^{* *}$. The first letter in dimer notation is the name of the amino acid where the proton is.

\begin{tabular}{lrrrrr} 
Conf. & $E$ & $G_{300}$ & $p_{300}$ & $G_{100}$ & $p_{100}$ \\
\hline DD-A1 & 0.00 & 2.36 & 0.23 & 0.00 & 0.97 \\
DD-B1 & 5.05 & 0.00 & 0.59 & 2.99 & 0.03 \\
DD-B2 & 5.13 & 4.72 & 0.09 & 4.65 & 0.00 \\
DD-A2 & 7.73 & 8.89 & 0.01 & 7.61 & 0.00 \\
DD-B3 & 8.04 & 6.12 & 0.05 & 6.50 & 0.00 \\
DD-B4 & 9.56 & 8.53 & 0.02 & 9.11 & 0.00 \\
\hline DL-A1 & 0.00 & 0.00 & 0.37 & 0.00 & 0.90 \\
DL-B1 & 1.28 & 5.14 & 0.05 & 2.71 & 0.03 \\
DL-B2 & 3.39 & 1.60 & 0.19 & 2.48 & 0.05 \\
DL-B3 & 4.45 & 0.72 & 0.28 & 3.03 & 0.02 \\
DL-B4 & 5.38 & 8.03 & 0.01 & 6.44 & 0.00 \\
DL-B5 & 7.29 & 3.33 & 0.10 & 6.26 & 0.00
\end{tabular}

